performance, growth of various mammals and rates of growth of bacteria, fungi and viruses. A section dealing with the effects of light and ionizing radiation occupies the next fifty pages and this is followed by a forty page section, the contents of which are devoted to the effects of sound, vibration and impact. The effects of acceleration and gravity are described in a twenty page section primarily in relation to man. The effects of the gaseous atmosphere on plants and animals are considered in three distinct sections totalling 180 pages, dealing with high and low concentrations of oxygen and carbon dioxide; total environmental pressure and atmospheric pollutants. The effects of water and solutes are considered in separate sections (with a total of 100 pages) primarily in relation to plants and aquatic animals. The tenth section of forty pages is devoted to biological rhythms. The book has two appendixes which give corresponding scientific and common names for animals and plants.

This handbook provides within a single cover a very extensive range of quantitative biological data. It is inevitable in a book of this type that some of the limitations of the value of particular groups of data are not apparent. In general, however, references to the literature are extensive and the book provides an easy method of access to the literature of fields outside a reader's normal sphere of knowledge. Thus, while the handbook could with advantage be held in the libraries of biological research and teaching institutions, the individual worker will probably find it of limited value in his own field.

J. Ernsting

\section{LABORATORY BIOLOGY}

\section{An Experimental Approach to Biology}

By Robert G. Thomson and Peter Abramoff. Pp. xi +253. (San Franciseo and London: W. H. Freeman and Company, 1966.) $26 s$.

Tris laboratory guide contains protocols for thirty-five experiments in nine areas of biology, and it is clear that the authors are well aware of students' difficulties. An introductory section covers safety measures, cleaning glassware, preparing standard solutions, and the use of centrifuges and volumetric glassware, while appendixes deal with $p \mathrm{H}$ meters and colorimeters.

The book would prove useful to those planning sixth form and introductory university courses, although some caveats must be entered. The experiments on active transport through bags of frog's skin, and on the induction of ovulation by injecting frogs with pituitary extracts prepared from anaesthetized frogs, will require care if the spirit of the laws protecting animals is to be observed. Measuring the volume of gas produced by Elodea without further analysis gives misleading data on the production of oxygen-and to show that the gas turns pyrogallate brown merely demonstrates the presence of oxygen, not its concentration. A large water reservoir in potometers is an anachronism, and in any event transpiration is easy to estimate directly by weighing. Benedict's solution contains sodium carbonate, not bicarbonate.

These are errors which can be corrected in a second edition. The general standard of the book is high, and it can be recommended.

A. K. Thomas

\section{STATISTICAL PAPERS}

\section{The Selected Papers of E. S. Pearson}

(Issued by the Biometrika Trustees to celebrate his 30 years as Editor.) Pp. vi +327 . (London: Cambridge University Press, 1966.) 40s. net.

The journal Biometrika was founded by Karl Pearson, W. F. R. Weldon and Francis Galton in order that papers on the applications of statistical method to biology and allied subjects might be published speedily and without hindrance from the editors of journals not specifically designed to cover such topics. The journal grew in circulation over the years but, such was the authority of Pearson in the statistical world, it remained very largely a house-journal until his death. When his son, E. S. Pearson, took over as editor in 1936 the statistical climate as well as the journal were changing. The proliferation of statistical centres all over the world was then beginning, which resulted in an outpouring of research papers which has grown steadily in volume until the present day.

Biometrika, the statistical world generally, and the English statistical world in particular owe much to E. S. Pearson's devoted and conscientious editorship during this climactic period. As ruler over a journal, still at the present day pre-eminent in England, he was able to influence to some extent the thinking of his contributors, and if we have not yet abandoned the search for common sense in data in favour of the hunting of the mathematical snark this is in part due to his careful refereeing and editing.

Much of E. S. Pearson's own work was published in Biometrika and it is fitting that the trustees should issue some of these papers to mark his retirement from the editorship. Out of the twenty-one papers all but three were first printed in the journal, half of them since 1945 . A further volume, which will reflect his research activity in company with $J$. Neyman during the thirties, is promised in a short time. The papers republished in this present volume show Pearson's main interests over the years non-normality, bases for the tests of hypotheses, analyses of accident data, approximations to probability distributions using moments.

It was a happy thought of the trustees to accompany this present volume with a volume containing the joint Neyman-Pearson papers and, in consort with the University of California Press, a volume of Neyman's own papers. Statisticians the world over will be grateful for their enterprise. F. N. DAVID

\section{UNDERWATER SOUND}

\section{Ocean Acoustics}

Theory and Experiment in Underwater Sound. By Ivan Tolstoy and C. S. Clay. (Advanced Physics Monograph Series.) Pp. $\mathrm{x}+203$. (New York: McGraw-Hill Book Company, Inc.; Maidenhead: McGraw-Hill Publishing Company, Ltd., 1966.) 124s. $6 d$.

The rather few monographs in this field tend to lean strongly toward either applied mathematics or engineering. This short volume holds admirably to a middle course, balancing mathematical analysis against experimental knowledge, and the idealizations required for a coherent theory against the real inhomogeneities and fluctuations.

The theme is acoustic propagation in shallow and deep water; sources, receivers and (except in one chapter) signal processing are purposely given little attention. The main attack is by normal-mode theory, in terms of eigenfunctions and eigenvalues rather than of residues in the complex plane. Ray methods and the intermediate WKB approximation are, however, earefully related to normal modes, and are used frequently. Geophysical and oceanographic complexities of the medium are constantly emphasized as limitations on feasible theory and on comparisons with experiment. Many such comparisons are given, numerically or graphically, with both clarifying and cautionary effects. Citations from the literature. averaging thirty in a chapter, amplify mathematical details, extend the theory, and give experimental results. 